\title{
Divergent Detonation Wave Processes in PBX Based on RDX
}

\author{
Ricardo A. L. Mendes*, José L. S. de A. Campos, Igor Y. Plaksin and Jose M. B. M. Ribeiro
}

LEDAP - Laboratory of Energetics and Detonics, Mechanical Eng. Dept., Fac. of Sciences and Technology of the University of Coimbra, Pinhal de Marrocos, Polo II, 3030-201 Coimbra (Portugal)

\begin{abstract}
In order to characterize the initial phase of the divergent detonation wave in PBX, a hemispheric explosive sample was initiated by a long cylindrical charge of the same explosive. The tested PBX is composed of $85 \mathrm{wt} \%$ of RDX and $15 \mathrm{wt} \%$ of binder based on HTPB. This PBX-RDX presents an effective density of $1.57 \mathrm{~g} / \mathrm{cm}^{3}$, and a detonation velocity of $7.90 \mathrm{~mm} / \mu \mathrm{s}$.

An optical method, based on ribbon consisting of 64 optical fibers (each fiber of $250 \mu \mathrm{m}$ diameter) and connected to a fast electronic streak camera, allows the measurement of the detonation velocity in the PBX hemisphere as a function of the run distance $R$ along several directions characterized by different angles with the axis of the sample.

The obtained results, presented as radius-time and velocity-radius functions, show the evolution of a divergent detonation wave in its run up to a radius equal to $33-34 \mathrm{~mm}(6.6-6.8$ times more than the diameter of the initiator). The real shape of the divergent detonation wave was obtained, and it was found significantly different from the ideal Huygens model.

The measurements of the pressure of a shock wave induced either by the divergent detonation wave or by a positive constant curvature detonation wave (detonation in a long cylindrical PBX charge) in an inert standard barrier show the existence of significant differences between them which can reach values up to 2.8 times less, in their amplitudes.
\end{abstract}

Keywords: Initiation, Divergent Detonation wave, PBX-RDX

\section{Introduction}

When a bulk explosive mass is directly initiated by a long cylindrical charge, the spherical divergent detonation wave (DW) formed in the bulk mass is characterized by detonation parameters, namely detonation velocity $[1-4]$ and detonation pressure [4-6], that are different from those observed in the normal detonation propagation in long cylindrical charges.

It was observed that in a hemisphere explosive sample, initiated in the central zone, the divergent DW reaches the periphery surface of the explosive sample quicker for some directions, characterized by an angle $\theta$ formed with the axis of the initiation charge, than for others $[7,8]$. It was also found that the value of this angle $\theta$ is a function of the explosive density [9], decreasing when its density increases. Furthermore, from what was experimentally observed it is

* Corresponding author; e-mail: ricardo.mendes@dem.uc.pt possible to say that for both, RDX and HMX based PBX samples, the propagation process of the divergent DW is a complex presenting a different non-monotonic behavior for each of the main directions of propagation from which results a significant delay in the arriving time of the DW to the periphery surface, for the directions making ninety or zero degrees with the axial axis of the initiation charge compared to the arriving time for the intermediated directions $[9-13,15]$. In the corner turning experiments, with $\mathrm{Cu}$ or PMMA confinement, it was shown that for the first $5 \mathrm{~mm}$ of the radial propagation the divergent detonation front presents an irregular shape with preferential acceleration directions $[12,13]$. All these results were obtained using a high-resolution optical method, developed at LEDAP, based on 64 optical fibers, with $250 \mu \mathrm{m}$ diameter each, that allows the almost continuous registrations of DW propagation [10-15].

Among other contributions to the study and understanding of the phenomena of the divergent DW propagation in PBX based on RDX that can be taken from this work, the most important is the experimental evidence of the great scale difference between the pressure amplitude in the divergent DW and the DW propagation in a cylindrical charge, with constant curvature.

\section{Experimental Setup and Results}

The tested PBX was composed of $85 \mathrm{wt} \%$ of RDX (bimodal particle distribution, $75 \mathrm{wt} \% \mathrm{~d}_{50}=96 \mu \mathrm{m}$ and $\left.25 \mathrm{wt} \% \mathrm{~d}_{50}=22 \mu \mathrm{m}\right)$ and $15 \mathrm{wt} \%$ of binder based on $\mathrm{HTPB}$, and presented an effective density of $1.57 \mathrm{~g} / \mathrm{cm}^{3}$ (>99\% TMD). The value of detonation velocity $D_{n}$, measured in long cylindrical charges, was equal to $7.90 \mathrm{~mm} / \mu \mathrm{s}$.

The hemispherical charges of PBX were initiated by long cylindrical charges with $5 \mathrm{~mm}$ of diameter filled with the same explosive. The diameter of the initiation charge exceeded 1.3 times the critical diameter of PBX, but it was smaller than the limit diameter of this composition. The values of critical and limit diameters, for the studied PBX, are equal to $3.75 \mathrm{~mm}$ and $10-15 \mathrm{~mm}$, respectively, and were determined measuring the detonation velocity in long cylindrical charges [11]. 
The velocities and the front shape of the divergent DW have been measured using a experimental setup shown schematically in Figure 1.

It comprises eleven sets with $5-6$ optical fibers covered by non-transparent film and oriented toward the center of the hemispherical charge. For several different radial distances in each set of the optical fibers a slit in the non-transparent film that was covering them was opened. For this reason, the light radiated by the detonation front (DF) when it crosses each of the slits is captured and transmitted by the fibers to an electronic streak camera where it is recorded on a photographic film.

A typical streak record of the divergent DW propagation in a PBX hemispheric charge, obtained with the already described experimental set-up is presented in Figure 2. It is possible to say from the analysis of this streak record that for each particular radial distance the arriving time is smaller for the propagation directions that makes an angle of $40^{\circ}-$ $50^{\circ}$ relatively to the axis of the charge.

Based on these experimental results, it was possible to evaluate the front shape of the divergent DW at several different time values (instants) i.e. the DF isochrones. The experimentally obtained isochrones, and the ones obtained with the ideal Huygens wave model are shown for comparison in Figure 3.

The presented results show a great deviation between the experimental divergent DW isochrones and the ideal Huygens one, that can reach, in the equatorial zone, up to $30 \%$ of difference. From this evidence it can be concluded, that, at least for the initial phase of its propagation ( $\mathrm{R}<10-20 \mathrm{~mm}$ ), the Huygens model is not acceptable to describe the process of the divergent DW propagation in PBX.

In reality, the shape of the DW front is almost quasispherical with some peculiarities on the equatorial zone. The center of this quasi-sphere is not located in the center of hemispherical bulk charge but inside of the bulk charge,

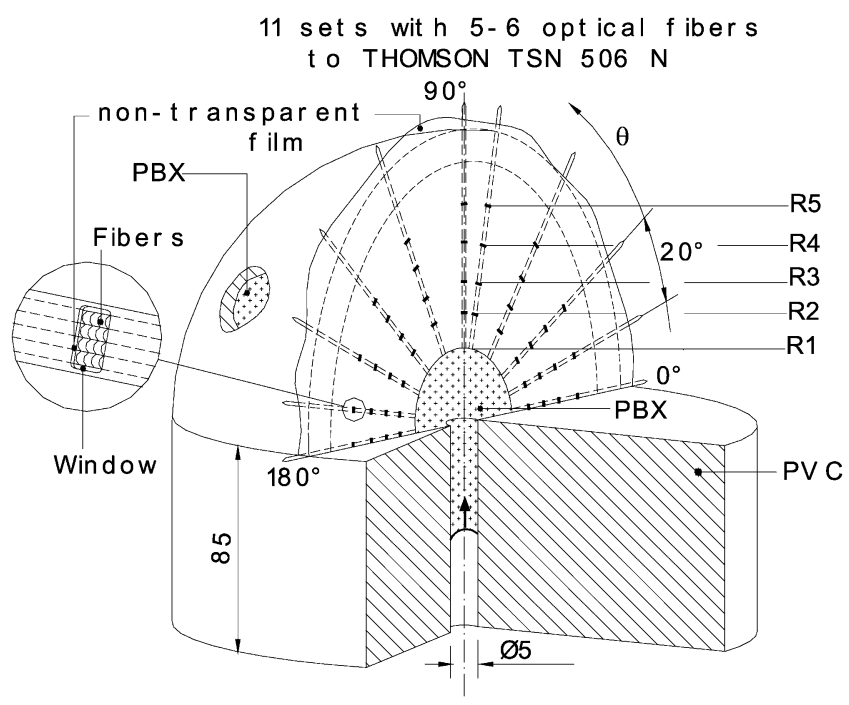

Figure 1. Experimental setup to characterize the shape and detonation velocity of the divergent detonation wave.

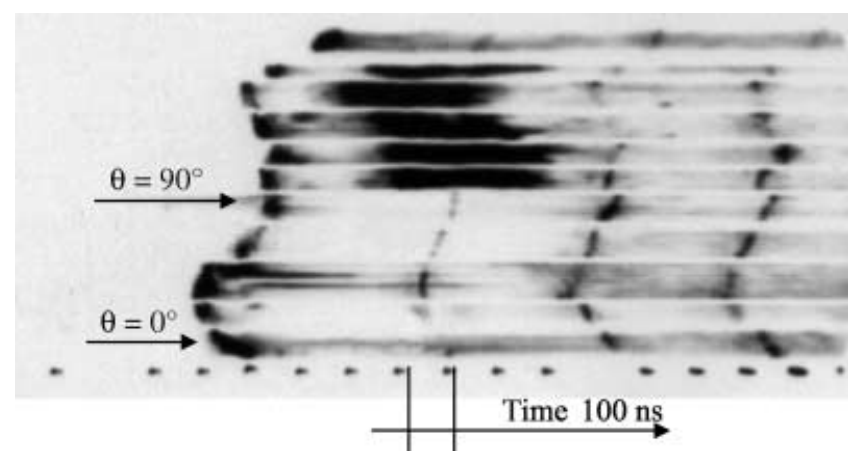

Figure 2. Typical streak camera record obtained with the experimental setup shown in Figure 1.

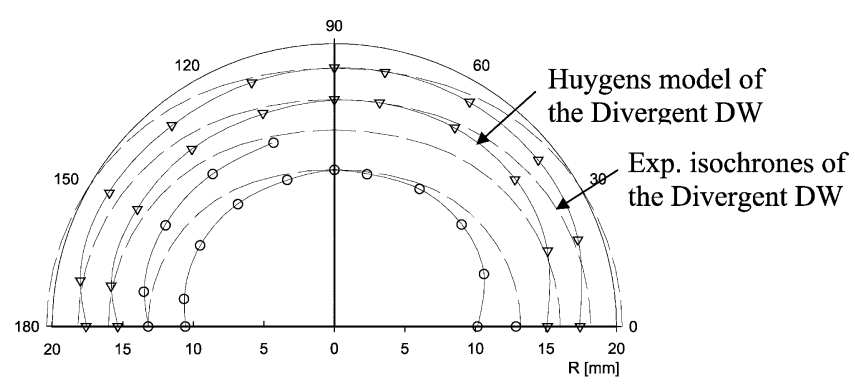

Figure 3. Experimental isochrones of the divergent DF (solid lines) and isochrones obtained with the Huygens model (dashed lines).

similar to what was observed by other authors in the earlier published work [8].

Knowing the distance between each of the slits opened in the fiber covered and measuring the time intervals between the successive light signals in the streak record it was possible to evaluate the divergent DW propagation velocity $D_{d}$, in the different DF zones, along each of the predefined radial directions (coincident with the orientation of the sets of the optical fibers). The obtained values of $D_{d}$, divided by the value of the detonation velocity in the cylindrical long charges $D_{n}=7.90 \mathrm{~mm} / \mu \mathrm{s}$, are presented as a function of the radial distance $(\mathrm{R}$ is taken from the center of the hemispherical PBX charges) for the directions of $0^{\circ}, 40^{\circ}$ and $90^{\circ}$ as shown in Figure 4.

Beyond the observed expected differences in the detonation velocity values, at fixed radial distances for the different directions of propagation, the results show for each of these directions a non-monotonous increase of DF velocity values. That means that the development of the divergent DW inside the bulk part of PBX, when it is initiated directly by long cylindrical charges of relatively small diameter, is made by consecutive phases of acceleration and slowing down.

In the earlier published works, the non-monotonous behavior of the divergent DW in PBX, at different radii of its propagation: $R=0-6 \mathrm{~mm}$ [12] and $R=0-15 \mathrm{~mm} \mathrm{[15],}$ has been recorded by a continuous method. One of the obtained results [10] that confirm the non-monotonous 


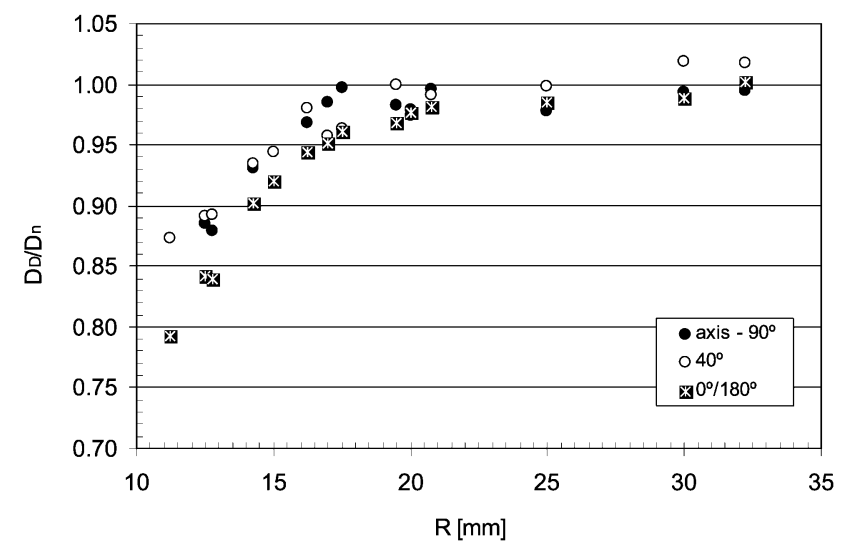

Figure 4. Velocities of the divergent detonation wave as a function of the radial distance for different $\theta$ angles.

(pulsing) development of the DW velocity, in two predefined directions, is presented in Figure 5.

The evaluation of the DW pressure in this work was made by an indirect way, measuring the intensity of the shock wave (SW) that is induced by the DW in an inert standard material (multi layered barrier). The pressure variation inside the inert barrier was evaluated in this work for two different phenomena, for the diverging and the constant curvature DW, which comprises the measurement in the central zone in two experimental configurations: the $\mathrm{PBX}$ hemispherical charge and the PBX long cylindrical charge (diameter $=10 \mathrm{~mm}$ ).

The multi-layered barrier consists of several layers of polymeric material that is organized in such a way that air micro gaps $(10 \mu \mathrm{m})$ are left between each two successive layers. When the SW crosses the gap, the shocked air generates light flashes, which allow the simultaneous determination of the position and time of propagation. By this way it is possible to evaluate the mean value of the shock wave velocity in each layer. The shock wave pressure is then evaluated using the shock Hugoniot data for the standard material. The standard material used for this experiments was the Polyimide Kapton in layers of $125 \mu \mathrm{m}$ thickness each.

This multi-layered barrier is placed in direct contact with the external surface of the explosive bulk charge as it is

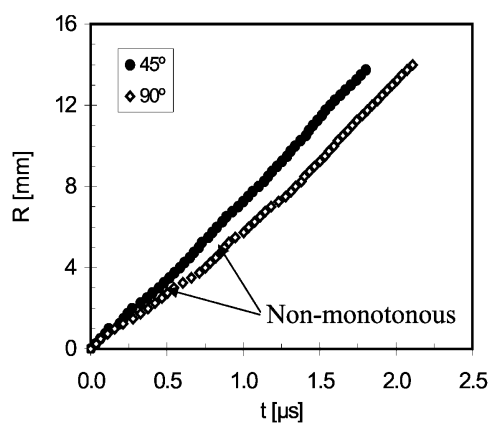

Figure 5. Non-monotonous (pulsing) development of the divergent DF in PBX based on RDX registered under two directions of propagation [10]: $45^{\circ}$ and $90^{\circ}$. shown schematically in Figure 6. Eleven sets of 5 to 6 optical fibers are then placed after this barrier, oriented toward the center of the charge, at different angles $\theta$ with its axis. The experimental setup allows, in the same experiment, the evaluation of the pressure induced $P_{\text {ind }}$ in the inert barrier by the diverging DW as a function of the propagation angle $\theta$.

The typical photo-chronogram obtained with the experimental setup described and shown in Figure 6 is presented in Figure 7.

In the graphic of Figure 8 the variation of the pressure values of the SW is shown induced by the divergent DW in the inert barrier as a function of the propagation distance, for two radii of the hemispherical PBX bulk charge $R_{s p h}=$ $15 \mathrm{~mm}$ and $R_{s p h}=20 \mathrm{~mm}$, and for a long PBX cylindrical charge with a diameter of $10 \mathrm{~mm}$. The results show that in the cases of the divergent DW, the pressure induced in the inert barrier increases with the radius of PBX hemispherical charge. But, despite of the fact that the radius of the hemispherical charge is more than two times bigger than the radius of the long cylindrical charge, the induced SW pressure values are 1.9-2.8 times smaller.

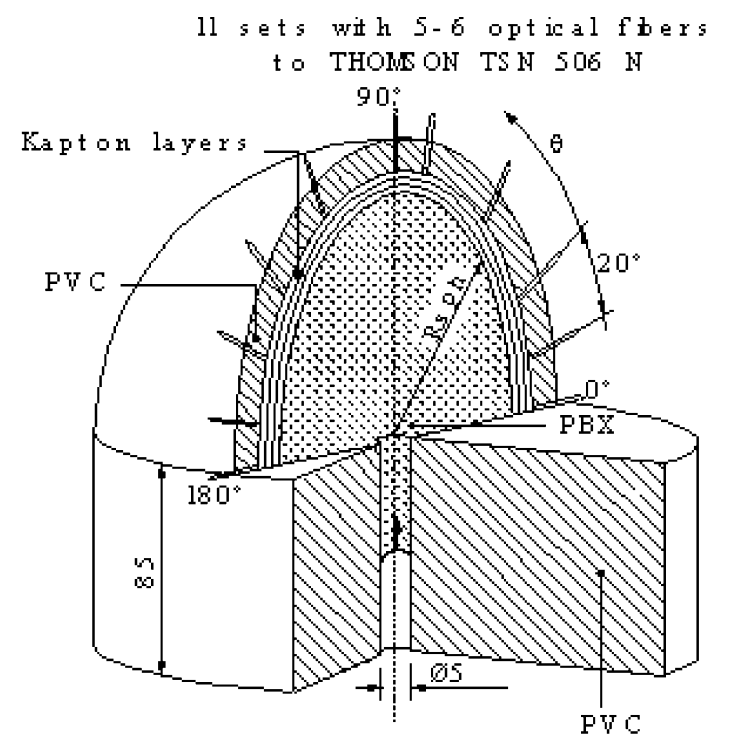

Figure 6. Experimental setup to measure the induced pressure in an inert barrier.

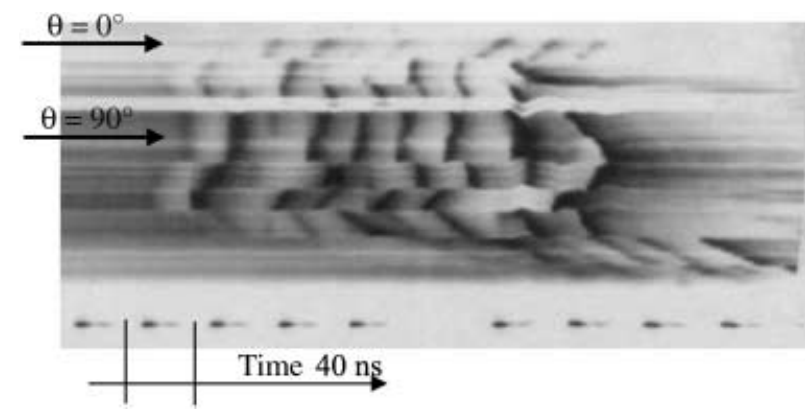

Figure 7. Shock wave propagation, inside the inert multi-layer barrier, induced by the divergent DW. 


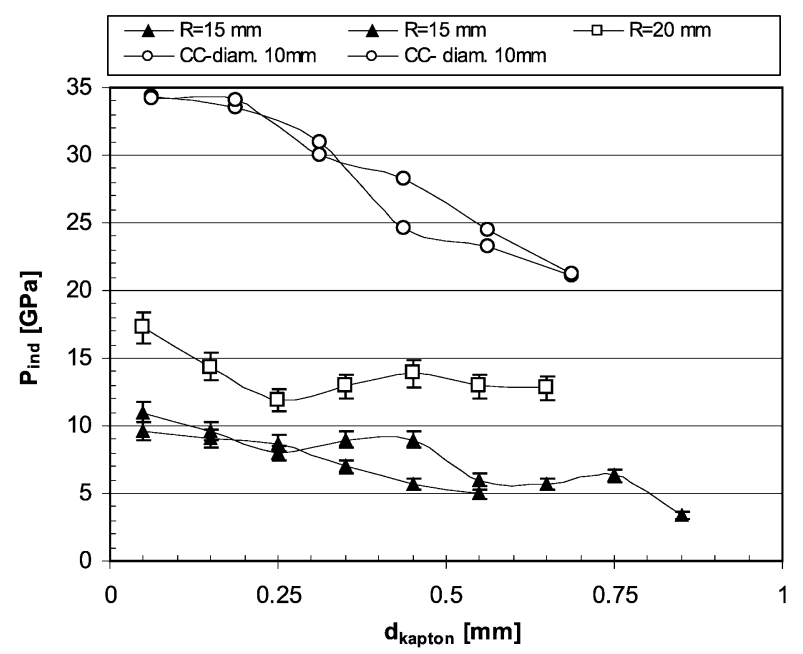

Figure 8. Pressure induced on barrier by a divergent DW and by a positive curvature DW (CC-cylindrical charge).

The most significant results that can be taken from these measurements are related with the scale of the differences observed between the values of the DW velocity and also between the values of the induced SW pressure that were obtained for the two tested geometries. The scale of this differences is in the order of $4.5 \%$ for $R_{s p h}=15 \mathrm{~mm}$ and $1.5 \%$ for $R_{s p h}=20 \mathrm{~mm}$ to the DW velocity and in the order of $65 \%$ for $R_{s p h}=15 \mathrm{~mm}$ and $45 \%$ for $R_{s p h}=20 \mathrm{~mm}$ for the induced pressure.

This scale of differences, in detonation velocity and induced pressure, between constant positive curvature and divergent DW in PBX obtained experimentally is in agreement with the results of the earlier published paper [4].

\section{Conclusions}

The development process of a divergent DW, in the PBXRDX hemispherical charges, initiated directly by a long cylindrical PBX-RDX charge of small diameter $(\phi=5 \mathrm{~mm})$, has been studied using a high-resolution optical method based on the application of the 64 optical fiber strip.

The obtained results show a big deviation between the experimental shape of the divergent DW and the one predicted by the Huygens model, considering a sphere with its center coincident with the center of the hemispheric PBX charge, the divergent DW presents, in the axial direction and in the equatorial direction, a propagation delay relatively to the intermediate directions.

The results confirm the non-monotonous development of the divergent DW in PBX-RDX under the applied conditions of initiation.

Whereas the difference in the values of PBX detonation velocity, observed between the values obtained in the long cylindrical charge and the values obtained for the axial direction, in the divergent DW during the first $20 \mathrm{~mm}$ of its propagation, reaches no more than $4.5 \%$, the scale of difference observed for the SW pressure induced in an inert standard barrier, for the same about dimensions of the hemispherical PBX charges, is significant, about $45 \%$ to $60 \%$.

\section{References}

[1] R. Cheret, G. Verdes, Divergent Spherical Detonation Waves in a Solid Explosive, 5th Symposium (International) on Detonation, Pasadena, CA, August 18-21, 1970, pp. 31.

[2] F. Bonthoux, P. Deneuville, and Y. Longueville, Diverging Detonations in RDX and PETN based Cast-cured PBX, 7th Symposium (International) on Detonation, Annapolis, MD, June 16-19, 1981, pp. 408.

[3] C. L. Mader, Numerical Modelling of Detonation, University of California Press, Berkley, 1979.

[4] V. M. Gerasimov, V. A. Gubachev, V. A. Vakin, I. E. Plaksin, and V. I. Shutov, Sov. J. Chem. Phys. 1994, 12, 1091.

[5] C. Tarver, P. A. Urtiev, Progr. In Astronautic and Aeronaut. 1984, 94, 369.

[6] M. V. Titov, S. A. Karakhanov, and S. A. Bordzilovsky, Pressure Variation Upon Iinitiation of a Cast RDX/TNT 50/ 50 Charge by Diverging Shock Wave, 8th Symposium (International) on Detonation, Albuquerque, NM, July 15 19, 1985, pp. 143.

[7] M. Cox, A. W. Campbell, Corner Turning in TATB, 7th Symposium (International) on Detonation, Annapolis, MD, June 16-19, 1981, pp. 624.

[8] M. Held, Influence of the Initiation Intensity on the Radial Detonation Breakthrough, Propellants, Explos., Pyrotech. 1995, 20, 245.

[9] L. G. Hill, C. A. Seitz, C. A. Forest, and H. H. Harry, High Explosive Corner Turning Performance and the LANL Mushroom Test, in S. C. Schmidt et al. (Eds.), Shock Compression of Condensed Matter, AIP Conference Proceedings 429, New York 1998, pp. 751.

[10] R. Mendes, I. Plaksin, and J. Campos, Single and Two Initiation Points of PBX, in S. C. Schmidt et al. (Eds.), Shock Compression of Condensed Matter, AIP Conference Proceedings 429, New York 1998, pp. 715.

[11] R. Mendes, Iniciação e Detonação de Explosivo Plástico de $R D X, \mathrm{PhD}$. Thesis, University of Coimbra, Portugal, 2002.

[12] I. Plaksin, J. Campos, R. Mendes, M. Mendonça and J. Gois, Interaction of Double Corner turning Effect in PBX, in Schmidt et al. (Eds.), Shock Waves in Compressed Matter, AIP Conference Proceedings 429, American Physics Institute, New York, 1998, pp. 755.

[13] I. Plaksin, J. Campos, R. Mendes, J. Ribeiro and J. Góis, Pulsing Behaviour and Corner Tturning Effect in PBX, 11th Symposium (International) on Detonation, Snowmass Village, Colorado, August 31 -September 4, 1998, pp. 679.

[14] M. Mendonça, I. Plaksin, J. Campos, and J. Góis, Emulsion Explosives with TNT, Chem. Phys. Reports, 1998, 17, 137.

[15] R. Mendes, I. Plaksin and J. Campos, Double Initiation and Mach Formation in PBX, 11th Symposium (International) on Detonation, Snowmass Village, Colorado, August 31September 4, 1998, pp. 399.

(Received December 16, 2002; Ms 2002/057) 